\title{
Nonlinear problem with subcritical exponent in Sobolev space
}

lqbal H Jebril ${ }^{*}$

\section{"Correspondence:}

iqbal501@hotmail.com

Department of Mathematics, Taibah

University, 344 Almadinah

Almunawwarah, Saudi Arabia

\section{Abstract}

Using Brouwer's fixed point theorem, we prove the existence of solutions for some nonlinear problem with subcritical Sobolev exponent in $S_{+}^{4}$.

MSC: Primary 46E35; 47H10; secondary 35J60

Keywords: Sobolev spaces; subcritical exponent; nonlinear problem

\section{Introduction and the main result}

The exponent Lebesgue space $L^{p}(\Omega)$ is defined by

$$
L^{p}(\Omega)=\left\{u \in L_{\mathrm{loc}}^{1}(\Omega): \int_{\Omega}|u(x)|^{p} d x<\infty\right\} .
$$

This space is endowed with the norm

$$
\|u\|_{L^{p}(\Omega)}=\inf \left\{\lambda>0: \int_{\Omega}\left|\frac{u(x)}{\lambda}\right|^{p} d x \leq 1\right\} .
$$

The Sobolev space $W^{1, p}(\Omega)$ is defined by

$$
W^{1, p}(\Omega)=\left\{u \in W_{\text {loc }}^{1,1}(\Omega): u \in L^{p}(\Omega) \text { and }|\nabla u| \in L^{p}(\Omega)\right\} .
$$

The corresponding norm for this space is

$$
\|u\|_{W^{1, p}(\Omega)}=\|u\|_{L^{p}(\Omega)}+\|\nabla u\|_{L^{p}(\Omega)} .
$$

Define $W_{0}^{1}(\Omega)=H_{0}^{1}(\Omega)$ as the closure of $C_{c}^{\infty}(\Omega)$ with respect to the $W^{1, p}(\Omega)$ norm which is a Hilbert space [1].

We consider the problem of the scalar curvature on the standard four dimensional half sphere under minimal boundary conditions $(S)$ :

(S) $\left\{\begin{array}{lll}L_{g} u:=-\Delta_{g} u+2 u=K u^{3}, & u>0 & \text { in } S_{+}^{4}, \\ \frac{\partial u}{\partial v}=0 & \text { on } \partial S_{+}^{4},\end{array}\right.$

(c) The Author(s) 2016. This article is distributed under the terms of the Creative Commons Attribution 4.0 International License (http://creativecommons.org/licenses/by/4.0/), which permits unrestricted use, distribution, and reproduction in any medium, provided you give appropriate credit to the original author(s) and the source, provide a link to the Creative Commons license, and indicate if changes were made. 
where $S_{+}^{4}=\left\{x \in \mathbb{R}^{5} /|x|=1, x_{5}>0\right\}, g$ is the standard metric, and $K$ is a $C^{3}$ positive Morse function on $\overline{S_{+}^{4}}$.

The scalar curvature problem on $S^{n}$ and also on $S_{+}^{n}$ was the subject of several works in recent years, we can cite for example [2-12].

Recall that the embedding of $H^{1}\left(S_{+}^{4}\right)$ into $L^{4}\left(S_{+}^{4}\right)$ is noncompact. For this reason, we have focused our study on the family of subcritical problems $\left(S_{\varepsilon}\right)$

$$
\left(S_{\varepsilon}\right)\left\{\begin{array}{lll}
-\Delta_{g} u+2 u=K u^{3-\varepsilon}, & u>0 & \text { in } S_{+}^{4}, \\
\frac{\partial u}{\partial v}=0 & \text { on } \partial S_{+}^{4},
\end{array}\right.
$$

where $\varepsilon$ is a small positive parameter.

Note that the solutions of problem $(S)$ can be the limit as $\varepsilon \rightarrow 0$ of some solutions $\left(u_{\varepsilon}\right)$ for $\left(S_{\varepsilon}\right)$.

Djadli et al. [13] studied this problem in the case of the three dimensional half sphere. Assuming that the critical points of $K_{1}$ verify $(\partial K / \partial v)\left(a_{i}\right)>0$ they demonstrated that there exist solutions $\left(u_{\varepsilon}\right)$ concentrated at the points $\left(a_{1}, \ldots, a_{p}\right)$. Moreover, in [14], we established the existence of another type of solutions $\left(u_{\varepsilon}\right)$ of $\left(S_{\varepsilon}\right)$ such that is concentrated at two points $a_{1} \in \partial S_{+}^{4}$ and $a_{2} \in S_{+}^{4}$.

In this work, we aim to construct some positive solutions of $\left(S_{\varepsilon}\right)$ which are concentrated at two different points of the boundary. To state our result, we will give the following notations. For $a \in \overline{S_{+}^{4}}$ and $\lambda>0$, let

$$
\delta_{(a, \lambda)}(x)=c_{0} \frac{\lambda}{\left(\lambda^{2}+1+\left(1-\lambda^{2}\right) \cos d(a, x)\right)},
$$

where $d$ is the geodesic distance on $\left(\overline{S_{+}^{4}}, g\right)$ and $c_{0}$ is chosen so that $\delta_{(a, \lambda)}$ is the family of solutions of the following problem:

$$
-\Delta u+2 u=u^{3}, \quad u>0, \quad \text { in } S^{4} .
$$

The space $H^{1}\left(S_{+}^{4}\right)$ is equipped with the norm $\|\cdot\|$ and its corresponding inner product $\langle\cdot, \cdot\rangle$ :

$$
\|f\|^{2}=\int_{S_{+}^{4}}|\nabla f|^{2}+2 \int_{S_{+}^{4}} f^{2}, \quad \text { and } \quad\langle f, g\rangle=\int_{S_{+}^{4}} \nabla f \nabla g+2 \int_{S_{+}^{4}} f g, \quad f, g \in H^{1}\left(S_{+}^{4}\right) \text {. }
$$

Theorem 1 Let $z_{1}$ and $z_{2}$ be a nondegenerate critical points of $K_{1}=K_{\mid \partial S_{+}^{4}}$ with $(\partial K / \partial v)\left(z_{i}\right)>$ $0, i=1,2$. Then there exists $\varepsilon_{0}>0$ such that, for each $\varepsilon \in\left(0, \varepsilon_{0}\right)$, problem $\left(S_{\varepsilon}\right)$ has a solution $\left(u_{\varepsilon}\right)$ of the form

$$
u_{\varepsilon}=\alpha_{1} \delta_{\left(x_{1}, \lambda_{1}\right)}+\alpha_{2} \delta_{\left(x_{2}, \lambda_{2}\right)}+v,
$$

where, as $\varepsilon \rightarrow 0, \alpha_{i} \rightarrow K\left(z_{i}\right)^{-1 / 2} ;\|v\| \rightarrow 0 ; x_{i} \rightarrow z_{i} ; x_{i} \in \partial S_{+}^{4} ; \lambda_{i} \rightarrow+\infty ; \lambda_{1}=c \lambda_{2}(1+o(1))$.

The rest of this work is summarized as follows. In Section 2, we present a classical preliminaries and we perform a useful estimations of functional $\left(I_{\varepsilon}\right)$ associated to the problem $\left(S_{\varepsilon}\right)$ for $(\varepsilon>0)$ and its gradient. Section 3 is devoted to the construction of solutions and the proof of our result. 


\section{Useful estimations}

We introduce the structure variational associated to the problem $\left(S_{\varepsilon}\right)$ for $\varepsilon>0$

$$
I_{\varepsilon}(u)=\frac{1}{2} \int_{S_{+}^{4}}|\nabla u|^{2}+\int_{S_{+}^{4}} u^{2}-\frac{1}{4-\varepsilon} \int_{S_{+}^{4}} K|u|^{4-\varepsilon}, \quad u \in H^{1}\left(S_{+}^{4}\right) .
$$

It is well known that there is an equivalence between the existence of solutions for $\left(S_{\varepsilon}\right)$ and the positive critical point of $I_{\varepsilon}$. Moreover, in order to reduce our problem to $\mathbb{R}_{+}^{4}$ we will perform some stereographic projection. We denote $D^{1,2}\left(\mathbb{R}_{+}^{4}\right)$ for the completion of $C_{c}^{\infty}\left(\overline{\mathbb{R}_{+}^{4}}\right)$ with respect to the Dirichlet norm. Recall that an isometry $1: H^{1}\left(S_{+}^{4}\right) \rightarrow D^{1,2}\left(\mathbb{R}_{+}^{4}\right)$ is induced by the stereographic projection $\pi_{a}$ about a point $a \in \partial S_{+}^{4}$ following the formula

$$
(1 \phi)(y)=\left(\frac{2}{1+|x|^{2}}\right) \phi\left(\pi_{a}^{-1}(y)\right), \quad \phi \in H^{1}\left(S_{+}^{4}\right), y \in \mathbb{R}_{+}^{4} .
$$

For every $\phi \in H^{1}\left(S_{+}^{4}\right)$, one can check that the following holds true:

$$
\int_{S_{+}^{4}}\left(|\nabla \phi|^{2}+2 \phi^{2}\right)=\int_{\mathbb{R}_{+}^{4}}|\nabla(1 \phi)|^{2} \quad \text { and } \quad \int_{S_{+}^{4}}|\phi|^{4}=\int_{\mathbb{R}_{+}^{4}}|1 \phi|^{4} .
$$

Furthermore, using (3) with $\pi_{-a}$, it is easy to see that $1 \delta_{(a, \lambda)}$ is given by

$$
{ }_{1} \delta_{(a, \lambda)}=\frac{c_{0} \lambda}{1+\lambda^{2}|x-a|^{2}} .
$$

$\delta_{(a, \lambda)}$ will be written instead of $1 \delta_{(a, \lambda)}$ in the sequel.

Let

$$
\begin{aligned}
M_{\varepsilon}= & \left\{m=(\alpha, \lambda, x, v) \in \mathbb{R}^{2} \times\left(\mathbb{R}_{+}^{*}\right)^{2} \times\left(\partial S_{+}^{4}\right)^{2} \times H^{1}\left(S_{+}^{4}\right): v \in E_{(x, \lambda)},\|v\|<v_{0} ;\right. \\
& \left|\frac{\alpha_{i}^{2} K\left(x_{i}\right)}{\alpha_{j}^{2} K\left(x_{j}\right)}-1\right|<v_{0}, \lambda_{i}>\frac{1}{\nu_{0}}, \varepsilon \log \lambda_{i}<v_{0}, \forall i ; c_{0}<\frac{\lambda_{1}}{\lambda_{2}}<c_{0}^{-1} ;\left|x_{1}-x_{2}\right|>d_{0} ; \\
& \left.\left|-2 c_{3} \frac{\partial K}{\partial \nu}\left(x_{i}\right) \frac{1}{\lambda_{i}}+\frac{\varepsilon K\left(x_{i}\right) S_{4}}{8}\right|<\varepsilon^{1+\frac{\sigma}{2}}\right\},
\end{aligned}
$$

where $v_{0}$ is a small positive constant, $\sigma, c_{0}, d_{0}$ are some suitable positive constants, and

$$
E_{(x, \lambda)}=\left\{w \in H^{1}\left(S_{+}^{4}\right) /\langle w, \varphi\rangle=0 \forall \varphi \in \operatorname{Span}\left\{\delta_{i}, \frac{\partial \delta_{i}}{\partial \lambda_{i}}, \frac{\partial \delta_{i}}{\partial x_{i}^{j}}, i=1,2 ; j \leq 4\right\}\right\} .
$$

Here, $x_{i}^{j}$ denotes the $j$ th component of $x_{i}$. Also

$$
\Psi_{\varepsilon}: M_{\varepsilon} \rightarrow \mathbb{R} ; \quad m=(\alpha, \lambda, x, v) \mapsto I_{\varepsilon}\left(\alpha_{1} \delta_{\left(x_{1}, \lambda_{1}\right)}+\alpha_{2} \delta_{\left(x_{2}, \lambda_{2}\right)}+v\right) .
$$

In the sequel, we will write $\delta_{i}$ instead of $\delta_{\left(x_{i}, \lambda_{i}\right)}$ and $u=\alpha_{1} \delta_{1}+\alpha_{2} \delta_{2}+v$ for the sake of simplicity.

In the remainder of this section, we will give expansions of the gradient of the functional $I_{\varepsilon}$ associated to $\left(S_{\varepsilon}\right)$ for $\varepsilon>0$. Thus estimations are needed in Section 3. We need to recall 
that [15] proved the following remark when $n=3$, but the same argument is available for the dimension 4 .

Remark 2 For $\varepsilon>0$ and $\delta_{(a, \lambda)}$ defined in (1), we have

$$
\delta_{(a, \lambda)}^{-\varepsilon}(x)=1-\varepsilon \log \delta_{(a, \lambda)}+O\left(\varepsilon^{2} \log ^{2} \lambda\right) \quad \text { in } S_{+}^{4} .
$$

Now, explicit computations, using Remark 2, yield the following propositions.

Proposition 3 Let $(\alpha, \lambda, x, v) \in M_{\varepsilon}$. Then, for $u=\alpha_{1} \delta_{\left(x_{1}, \lambda_{1}\right)}+\alpha_{2} \delta_{\left(x_{2}, \lambda_{2}\right)}+v$, we have the following expansion:

$$
\left\langle\nabla I_{\varepsilon}(u), \delta_{i}\right\rangle=\frac{\alpha_{i} S_{4}}{2}\left(1-\alpha_{i}^{2-\varepsilon} K\left(x_{i}\right)\right)+O\left(\varepsilon \log \lambda_{i}+\frac{1}{\lambda_{i}}+\varepsilon_{12}+\|v\|^{2}\right),
$$

where

$$
\begin{aligned}
\varepsilon_{i j} & =\frac{1}{\frac{\lambda_{i}}{\lambda_{j}}+\frac{\lambda_{j}}{\lambda_{i}}+\lambda_{i} \lambda_{j}\left|a_{i}-a_{j}\right|^{2}}, \\
S_{4} & =64 \int_{\mathbb{R}^{4}} \frac{d x}{\left(1+|x|^{2}\right)^{4}} .
\end{aligned}
$$

Proof We have

$$
\left\langle\nabla I_{\varepsilon}(u), h\right\rangle=\int_{S_{+}^{4}} \nabla u \nabla h+2 \int_{S_{+}^{4}} u h-\int_{S_{+}^{4}} K u^{3-\varepsilon} h .
$$

A computation similar to the one performed in [16] shows that, for $i=1,2$,

$$
\left\|\delta_{i}\right\|^{2}=\int_{\mathbb{R}_{+}^{4}}\left|\nabla \delta_{i}\right|^{2}=\frac{S_{4}}{2}
$$

and

$$
\int_{S_{+}^{4}} \nabla \delta_{i} \nabla \delta_{j}+2 \int_{S_{+}^{4}} \delta_{i} \delta_{j}=\int_{\mathbb{R}_{+}^{4}} \nabla \delta_{i} \nabla \delta_{j}=\int_{\mathbb{R}_{+}^{4}} \delta_{i}^{3} \delta_{j}=O\left(\varepsilon_{12}\right) .
$$

For the other integral, we write

$$
\int_{S_{+}^{4}} K u^{3-\varepsilon} \delta_{i}=\int_{S_{+}^{4}} K\left(\alpha_{1} \delta_{1}+\alpha_{2} \delta_{2}\right)^{3-\varepsilon} \delta_{i}+O\left(\varepsilon_{12}^{2} \log \varepsilon_{12}^{-1}+|v|^{2}\right) .
$$

We also write

$$
\begin{aligned}
\int_{S_{+}^{4}} K\left(\alpha_{1} \delta_{1}+\alpha_{2} \delta_{2}\right)^{3-\varepsilon} \delta_{i}= & \alpha_{i}^{3-\varepsilon} \int_{S_{+}^{4}} K \delta_{i}^{4-\varepsilon}+\alpha_{j}^{3-\varepsilon} \int_{S_{+}^{4}} K \delta_{j}^{3-\varepsilon} \delta_{i} \\
& +(3-\varepsilon) \alpha_{i}^{2-\varepsilon} \alpha_{j} \int_{S_{+}^{4}} K \delta_{i}^{3-\varepsilon} \delta_{j}+O\left(\varepsilon_{12}^{2} \log \varepsilon_{12}^{-1}\right) .
\end{aligned}
$$


Expansions of $K$ around $x_{i}$ and $x_{j}$ give

$$
\begin{aligned}
& \int_{S_{+}^{4}} K \delta_{i}^{4-\varepsilon}=\int_{\mathbb{R}_{+}^{4}} K \delta_{i}^{4-\varepsilon}=K\left(x_{i}\right) \frac{S_{4}}{2}+O\left(\varepsilon \log \lambda_{i}+\frac{1}{\lambda_{i}}\right), \\
& \int_{S_{+}^{4}} K \delta_{j}^{3-\varepsilon} \delta_{i}=\int_{\mathbb{R}_{+}^{4}} K \delta_{j}^{3-\varepsilon} \delta_{i}=O\left(\varepsilon \log \lambda_{i}+\varepsilon_{12}\right), \\
& \int_{S_{+}^{4}} K \delta_{i}^{3-\varepsilon} \delta_{j}=\int_{\mathbb{R}_{+}^{4}} K \delta_{i}^{3-\varepsilon} \delta_{j}=O\left(\varepsilon \log \lambda_{i}+\varepsilon_{12}\right) .
\end{aligned}
$$

Combining (5)-(12), we derive our proposition.

Proposition 4 Let $(\alpha, \lambda, x, v) \in M_{\varepsilon}$. Then, for $u=\alpha_{1} \delta_{\left(x_{1}, \lambda_{1}\right)}+\alpha_{2} \delta_{\left(x_{2}, \lambda_{2}\right)}+v$, we have

$$
\begin{aligned}
\left\langle\nabla I_{\varepsilon}(u), \lambda_{i} \frac{\partial \delta_{i}}{\partial \lambda_{i}}\right\rangle= & \alpha_{j}\left(1-\alpha_{j}^{2-\varepsilon} K\left(x_{j}\right)-\alpha_{i}^{2-\varepsilon} K\left(x_{i}\right)\right) c_{2} \lambda_{i} \frac{\partial \varepsilon_{12}}{\partial \lambda_{i}}+\alpha_{i}^{3-\varepsilon} \frac{\varepsilon S_{4} K\left(x_{i}\right)}{8} \\
& +\alpha_{i}^{3-\varepsilon} \frac{2 c_{3}}{\lambda_{i}} \frac{\partial K}{\partial v}\left(x_{i}\right)+O\left(\|v\|^{2}+\frac{1}{\lambda_{i}^{2}}+\varepsilon^{2} \log \lambda_{i}+\frac{\varepsilon \log \lambda_{i}}{\lambda_{i}}\right) \\
& +O\left(\varepsilon \varepsilon_{12}\left(\log \varepsilon_{12}^{-1}\right)^{1 / 2}+\varepsilon_{12}^{2} \log \varepsilon_{12}^{-1}+\frac{\varepsilon_{12}}{\lambda_{j}}\left(\log \varepsilon_{12}^{-1}\right)^{1 / 2}\right)
\end{aligned}
$$

where

$$
S_{4}=64 \int_{\mathbb{R}^{4}} \frac{d x}{\left(1+|x|^{2}\right)^{4}}, \quad c_{2}=64 \int_{\mathbb{R}^{4}} \frac{d x}{\left(1+|x|^{2}\right)^{3}}, \quad c_{3}=64 \int_{\mathbb{R}_{+}^{4}} \frac{x_{4}\left(|x|^{2}-1\right)}{\left(1+|x|^{2}\right)^{5}} d x
$$

Proof Observe that (see [16])

$$
\begin{aligned}
& \int_{\mathbb{R}_{+}^{4}} \nabla \delta_{i} \nabla\left(\lambda_{i} \frac{\partial \delta_{i}}{\partial \lambda_{i}}\right)=\int_{\mathbb{R}_{+}^{4}} \delta_{i}^{3} \lambda_{i} \frac{\partial \delta_{i}}{\partial \lambda_{i}}=0 \\
& \int_{\mathbb{R}_{+}^{4}} \nabla \delta_{j} \nabla\left(\lambda_{i} \frac{\partial \delta_{i}}{\partial \lambda_{i}}\right)=\int_{\mathbb{R}_{+}^{4}} \delta_{j}^{3} \lambda_{i} \frac{\partial \delta_{i}}{\partial \lambda_{i}}=\frac{1}{2} c_{2} \lambda_{i} \frac{\partial \varepsilon_{12}}{\partial \lambda_{i}}+O\left(\varepsilon_{12}^{2} \log \left(\varepsilon_{12}^{-1}\right)\right) .
\end{aligned}
$$

For the other part, we have the expansions of $K$ around $x_{i}$ and using Remark 2 ,

$$
\begin{aligned}
& \int_{\mathbb{R}_{+}^{4}} K \delta_{i}^{3-\varepsilon} \lambda_{i} \frac{\partial \delta_{i}}{\partial \lambda_{i}}=-\frac{\varepsilon S_{4} K\left(x_{i}\right)}{8}-\frac{2 c_{3}}{\lambda_{i}} \nabla K\left(x_{i}\right) e_{4}+O\left(\varepsilon^{2} \log \lambda_{i}+\frac{1}{\lambda_{i}^{2}}+\frac{\varepsilon}{\lambda_{i}}\right), \\
& \int_{\mathbb{R}_{+}^{4}} K P \delta_{j}^{3-\varepsilon} \lambda_{i} \frac{\partial \delta_{i}}{\partial \lambda_{i}}=K\left(x_{j}\right) \frac{1}{2} c_{2} \lambda_{i} \frac{\partial \varepsilon_{12}}{\partial \lambda_{i}}+O\left(\varepsilon \varepsilon_{12}\left(\log \left(\varepsilon_{12}^{-1}\right)\right)^{\frac{1}{2}}+\frac{1}{\lambda_{j}^{2}}\right) \\
& +O\left(\varepsilon_{12}^{2} \log \left(\varepsilon_{12}^{-1}\right)\right) \\
& (3-\varepsilon) \int_{\mathbb{R}_{+}^{4}} K \delta_{i}^{2-\varepsilon} \delta_{j} \lambda_{i} \frac{\partial \delta_{i}}{\partial \lambda_{i}}=K\left(x_{i}\right) \frac{1}{2} c_{2} \lambda_{i} \frac{\partial \varepsilon_{12}}{\partial \lambda_{i}}+O\left(\varepsilon \varepsilon_{12}\left(\log \left(\varepsilon_{12}^{-1}\right)\right)^{\frac{1}{2}}\right) \\
& +O\left(\varepsilon_{12}^{2} \log \left(\varepsilon_{12}^{-1}\right)+\frac{\varepsilon_{12}}{\lambda_{j}}\left(\log \left(\varepsilon_{12}^{-1}\right)\right)^{\frac{1}{2}}\right) .
\end{aligned}
$$

Combining (5), (13), (14), (15), (16), and (17), the proof follows. 
Proposition 5 Let $(\alpha, \lambda, x, v) \in M_{\varepsilon}$. Then, for $u=\alpha_{1} \delta_{\left(x_{1}, \lambda_{1}\right)}+\alpha_{2} \delta_{\left(x_{2}, \lambda_{2}\right)}+v$, we have the following expansion:

$$
\begin{aligned}
\left\langle\nabla I_{\varepsilon}(u), \frac{1}{\lambda_{i}} \frac{\partial \delta_{i}}{\partial x_{i}}\right\rangle= & \left(\alpha_{i} c_{4}\left(1-\alpha_{i}^{2-\varepsilon} K\left(x_{i}\right)\right)+\alpha_{i}^{3-\varepsilon} K\left(x_{i}\right) \varepsilon\left(c_{4} \log \lambda_{i}+c_{7}\right)\right. \\
& \left.+2 \alpha_{i}^{3-\varepsilon} \frac{c_{5}}{\lambda_{i}} \frac{\partial K}{\partial \nu}\left(x_{i}\right)\right) e_{4}+\alpha_{j}\left(1-\sum \alpha_{i}^{2-\varepsilon} K\left(x_{i}\right)\right) \frac{c_{2}}{\lambda_{i}} \frac{\partial \varepsilon_{12}}{\partial x_{i}} \\
& -2 \alpha_{i}^{3-\varepsilon} c_{5} \frac{\nabla_{T} K\left(x_{i}\right)}{\lambda_{i}}+O\left(\|v\|^{2}+\lambda_{j}\left|x_{1}-x_{2}\right| \varepsilon_{12}^{\frac{5}{2}}+\frac{\varepsilon \log \lambda_{i}}{\lambda_{i}}\left|\nabla_{T} K\left(x_{i}\right)\right|\right) \\
& +O\left(\varepsilon \varepsilon_{12}\left(\log \varepsilon_{12}^{-1}\right)^{\frac{1}{2}}+\varepsilon_{12}^{2} \log \varepsilon_{12}^{-1}+\frac{\varepsilon_{12}}{\lambda_{j}}\left(\log \varepsilon_{12}^{-1}\right)^{\frac{1}{2}}+\frac{1}{\lambda_{i}^{2}}+\varepsilon^{2} \log ^{2} \lambda_{i}\right),
\end{aligned}
$$

where

$$
c_{4}=132 \int_{\mathbb{R}_{+}^{4}} \frac{x_{4}}{\left(1+|x|^{2}\right)^{5}} d x, \quad c_{5}=16 \int_{\mathbb{R}^{4}} \frac{x_{4}^{2}}{\left(1+|x|^{2}\right)^{5}} d x .
$$

Proof We have

$$
\begin{aligned}
& \int_{\mathbb{R}_{+}^{4}} \nabla \delta_{i} \nabla\left(\frac{1}{\lambda_{i}} \frac{\partial \delta_{i}}{\partial x_{i}}\right)=\int_{\mathbb{R}_{+}^{4}} \delta_{i}^{3} \frac{1}{\lambda_{i}} \frac{\partial \delta_{i}}{\partial x_{i}}=c_{4} e_{4} \\
& \int_{\mathbb{R}_{+}^{4}} \nabla \delta_{j} \nabla\left(\frac{1}{\lambda_{i}} \frac{\partial \delta_{i}}{\partial x_{i}}\right)=\int_{\mathbb{R}_{+}^{4}} \delta_{j}^{3} \frac{1}{\lambda_{i}} \frac{\partial \delta_{i}}{\partial x_{i}}=\frac{1}{2} \frac{c_{2}}{\lambda_{i}} \frac{\partial \varepsilon_{12}}{\partial x_{i}}+O\left(\varepsilon_{12}^{2} \log \left(\varepsilon_{12}^{-1}\right)+\varepsilon_{12}^{\frac{5}{2}} \lambda_{j}\left|x_{1}-x_{2}\right|\right) .
\end{aligned}
$$

For the other part

$$
\begin{aligned}
\int_{\mathbb{R}_{+}^{4}} K \delta_{i}^{3-\varepsilon} \frac{1}{\lambda_{i}} \frac{\partial \delta_{i}}{\partial x_{i}}= & K\left(x_{i}\right) c_{4} e_{4}+2 \frac{c_{5}}{\lambda_{i}} \nabla K\left(x_{i}\right)-\varepsilon \log \lambda_{i} K\left(x_{i}\right) c_{4} e_{4} \\
& -\varepsilon K\left(x_{i}\right) c_{7} e_{4}+O\left(\frac{1}{\lambda_{i}^{2}}+\varepsilon^{2} \log ^{2} \lambda_{i}\right), \\
\int_{\mathbb{R}_{+}^{4}} K \delta_{j}^{3-\varepsilon} \frac{1}{\lambda_{i}} \frac{\partial \delta_{i}}{\partial x_{i}}= & K\left(x_{j}\right) \frac{1}{2} c_{2} \frac{1}{\lambda_{i}} \frac{\partial \varepsilon_{12}}{\partial a_{i}}+O\left(\varepsilon_{12}^{\frac{5}{2}} \lambda_{j}\left|x_{1}-x_{2}\right|\right) \\
& +O\left(\varepsilon_{12}^{2} \log \left(\varepsilon_{12}^{-1}\right)+\frac{1}{\lambda_{j}} \varepsilon_{12}\left(\log \left(\varepsilon_{12}^{-1}\right)\right)^{\frac{1}{2}}\right), \\
(3-\varepsilon) \int_{\mathbb{R}_{+}^{4}} K \delta_{i}^{2-\varepsilon} \delta_{j} \frac{1}{\lambda_{i}} \frac{\partial \delta_{i}}{\partial x_{i}}= & K\left(x_{i}\right) \frac{1}{2} c_{2} \frac{1}{\lambda_{i}} \frac{\partial \varepsilon_{12}}{\partial x_{i}}+O\left(\varepsilon_{12}^{\frac{5}{2}} \lambda_{j}\left|x_{1}-x_{2}\right|\right) \\
& +O\left(\varepsilon_{12}^{2} \log \left(\varepsilon_{12}^{-1}\right)+\frac{1}{\lambda_{i}} \varepsilon_{12}\left(\log \left(\varepsilon_{12}^{-1}\right)\right)^{\frac{1}{2}}\right) .
\end{aligned}
$$

Using (5), (18)-(22), our proposition follows.

\section{Construction of the solution}

The method of this type of theorem was followed first by Bahri, Li and Rey [17] when they studied an approximation problem of the Yamabe-type problem on domains. Many authors used this idea to construct some solutions to other problems. The method becomes standard. Here we will follow the idea of [17] and take account of the new estimates since 
we have an equation different from the one studied in [17]. From the idea of [17], using the coefficients of Euler-Lagrange, we obtain

Proposition 6 Let A point $m=(\alpha, \lambda, x, v) \in M_{\varepsilon}$ is a critical point of the function $\Psi_{\varepsilon}$ if and only if $u=\alpha_{1} \delta_{1}+\alpha_{2} \delta_{2}+v$ is a critical point of functional $I_{\varepsilon}$, which means the existence of some $(A, B, C) \in \mathbb{R}^{2} \times \mathbb{R}^{2} \times\left(\mathbb{R}^{4}\right)^{2}$ with the following:

$$
\begin{aligned}
& \left(E_{\alpha_{i}}\right) \frac{\partial \Psi_{\varepsilon}}{\partial \alpha_{i}}=0, \quad \forall i=1,2, \\
& \left(E_{\lambda_{i}}\right) \frac{\partial \Psi_{\varepsilon}}{\partial \lambda_{i}}=B_{i}\left(\frac{\partial^{2} \delta_{i}}{\partial \lambda_{i}^{2}}, v\right\rangle+\sum_{j=1}^{4} C_{i j}\left(\frac{\partial^{2} \delta_{i}}{\partial x_{i}^{j} \partial \lambda_{i}}, v\right), \quad \forall i=1,2, \\
& \left(E_{x_{i}}\right) \frac{\partial \Psi_{\varepsilon}}{\partial x_{i}}=B_{i}\left(\frac{\partial^{2} \delta_{i}}{\partial \lambda_{i} \partial x_{i}}, v\right\rangle+\sum_{j=1}^{4} C_{i j}\left(\frac{\partial^{2} \delta_{i}}{\partial x_{i}^{j} \partial x_{i}}, v\right), \quad \forall i=1,2, \\
& \left(E_{v}\right) \frac{\partial \Psi_{\varepsilon}}{\partial v}=\sum_{i=1,2}\left(A_{i} \delta_{i}+B_{i} \frac{\partial \delta_{i}}{\partial \lambda_{i}}+\sum_{j=1}^{4} C_{i j} \frac{\partial \delta_{i}}{\partial x_{i}^{j}}\right) .
\end{aligned}
$$

Now, by a careful study of equation $\left(E_{v}\right)$, we get the following.

Proposition 7 [12] For any $(\varepsilon, \alpha, \lambda, x)$ with $(\alpha, \lambda, x, 0) \in M_{\varepsilon}$, there exists a smooth map which associates $\bar{v} \in E_{(x, \lambda)}$ with $\|\bar{v}\|<\nu_{0}$ and equation (26) in the previous proposition is verified for some $(A, B, C) \in \mathbb{R}^{2} \times \mathbb{R}^{2} \times\left(\mathbb{R}^{4}\right)^{2}$. Such $a \bar{v}$ is unique, minimizes $\Psi_{\varepsilon}(\alpha, \lambda, x, v)$ with respect to $v$ in $\left\{v \in E_{(x, \lambda)} /\|v\|<v_{0}\right\}$, and

$$
\|\bar{\nu}\|=O\left(\varepsilon+\frac{1}{\lambda_{1}}+\frac{1}{\lambda_{2}}+\varepsilon_{12}\left(\log \varepsilon_{12}^{-1}\right)^{1 / 2}\right) .
$$

Proof of Theorem 1 Once $\bar{v}$ is defined by Proposition 7, we estimate the corresponding numbers $A, B, C$ by taking the scalar product in $H^{1}\left(S_{+}^{4}\right)$ of $\left(E_{v}\right)$ with $\delta_{i}, \partial \delta_{i} / \partial \lambda_{i}, \partial \delta_{i} / \partial x_{i}$ for $i=1,2$, respectively. So we get the following coefficients of a quasi-diagonal system:

$$
\begin{aligned}
& \int_{\mathbb{R}_{+}^{4}}\left|\nabla \delta_{i}\right|^{2}=\frac{S_{4}}{2} ; \quad \int_{\mathbb{R}_{+}^{4}} \nabla \delta_{1} \nabla \delta_{2}=O\left(\frac{1}{\lambda_{2} \lambda_{1}}\right) ; \quad \int_{\mathbb{R}_{+}^{4}} \nabla \delta_{i} \nabla \frac{\partial \delta_{i}}{\partial \lambda_{i}}=0 ; \\
& \int_{\mathbb{R}_{+}^{4}} \nabla \delta_{1} \nabla \frac{\partial \delta_{2}}{\partial \lambda_{2}}=O\left(\frac{1}{\lambda_{1} \lambda_{2}^{2}}\right), \quad \int_{\mathbb{R}_{+}^{4}} \nabla \delta_{2} \nabla \frac{\partial \delta_{1}}{\partial \lambda_{1}}=O\left(\frac{1}{\lambda_{1}^{2} \lambda_{2}}\right) ; \quad \int_{\mathbb{R}_{+}^{4}}\left|\nabla \frac{\partial \delta_{i}}{\partial \lambda_{i}}\right|^{2}=\frac{\Gamma_{1}}{2 \lambda_{i}^{2}} ; \\
& \int_{\mathbb{R}_{+}^{4}} \nabla \frac{\partial \delta_{1}}{\partial \lambda_{1}} \nabla \frac{\partial \delta_{2}}{\partial \lambda_{2}}=O\left(\frac{1}{\lambda_{1}^{2} \lambda_{2}^{2}}\right), \quad \int_{\mathbb{R}_{+}^{4}}\left|\nabla \frac{\partial \delta_{i}}{\partial x_{i}}\right|^{2}=\frac{\Gamma_{2}}{2} \lambda_{i}^{2} ; \quad \int_{\mathbb{R}_{+}^{4}} \nabla \delta_{i} \nabla \frac{\partial \delta_{i}}{\partial x_{i}}=O\left(\lambda_{1}\right) ; \\
& \int_{\mathbb{R}_{+}^{4}} \nabla \delta_{1} \nabla \frac{\partial \delta_{2}}{\partial x_{2}}=O\left(\frac{1}{\lambda_{1}}\right), \quad \int_{\mathbb{R}_{+}^{4}} \nabla \delta_{2} \nabla \frac{\partial \delta_{1}}{\partial x_{1}}=O\left(\frac{1}{\lambda_{2}}\right) ; \\
& \int_{\mathbb{R}_{+}^{4}} \nabla \frac{\partial \delta_{1}}{\partial x_{1}} \nabla \frac{\partial \delta_{2}}{\partial x_{2}}=\frac{n+2}{n-2} \int_{\mathbb{R}_{+}^{4}} \delta_{2}^{\frac{4}{n-2}} \nabla \frac{\partial \delta_{2}}{\partial x_{2}} \frac{\partial \delta_{1}}{\partial x_{1}}=O\left(\frac{1}{\lambda_{1}}\right),
\end{aligned}
$$

with $\left|x_{1}-x_{2}\right| \geq c>0$ and $\Gamma_{1}, \Gamma_{2}$ are positive constants.

We have also

$$
\left\langle\frac{\partial \Psi_{\varepsilon}}{\partial v}, \delta_{i}\right\rangle=\frac{\partial \Psi_{\varepsilon}}{\partial \alpha_{i}} ; \quad\left\langle\frac{\partial \Psi_{\varepsilon}}{\partial v}, \frac{\partial \delta_{i}}{\partial \lambda_{i}}\right\rangle=\frac{1}{\alpha_{i}} \frac{\partial \Psi_{\varepsilon}}{\partial \lambda_{i}} ; \quad\left\langle\frac{\partial \Psi_{\varepsilon}}{\partial v}, \frac{\partial \delta_{i}}{\partial x_{i}}\right\rangle=\frac{1}{\alpha_{i}} \frac{\partial \Psi_{\varepsilon}}{\partial x_{i}} .
$$


Using Propositions 3, some computations yield

$$
\frac{\partial \Psi_{\varepsilon}}{\partial \alpha_{i}}=-S_{4} \beta_{i}+V_{\alpha_{i}}(\varepsilon, \alpha, \lambda, x)
$$

with $\beta_{i}=\alpha_{i}-1 / K\left(z_{i}\right)^{\frac{1}{2}}$ and

$$
V_{\alpha_{i}}=O\left(\beta_{i}^{2}+\varepsilon \log \lambda_{i}+\frac{1}{\lambda_{i}}+\left|x_{i}-z_{i}\right|^{2}\right)
$$

In the same way, using Propositions 4, we get

$$
\frac{\partial \Psi_{\varepsilon}}{\partial \lambda_{i}}=\frac{1}{K\left(z_{i}\right)}\left(\frac{2 c_{3}}{\lambda_{i}^{2}} \frac{\partial K}{\partial v}\left(x_{i}\right)+\frac{\varepsilon K\left(x_{i}\right) S_{4}}{8 \lambda_{i}}\right)+V_{\lambda_{i}}(\varepsilon, \alpha, \lambda, x)
$$

where $c_{2}$ and $c_{3}$ are defined in Proposition 4 and

$$
V_{\lambda_{i}}=O\left[\frac{1}{\lambda_{i}}\left(\frac{1}{\lambda_{i}^{2}}+\varepsilon^{2} \log \lambda_{i}+\frac{\varepsilon \log \lambda_{i}}{\lambda_{i}}\right)+\left(|\beta|+\varepsilon+\left|x_{i}-z_{i}\right|^{2}\right)\left(\frac{\varepsilon}{\lambda_{i}}+\frac{1}{\lambda_{i}^{2}}\right)\right] .
$$

Lastly, using Propositions 5, we have

$$
\frac{\partial \Psi_{\varepsilon}}{\partial x_{i}}=-2 c_{5} \nabla_{T} K\left(x_{i}\right)+V_{x_{i}}(\varepsilon, \alpha, \lambda, x)
$$

where

$$
V_{x_{i}}=O\left(\frac{1}{\lambda_{i}}+\left(|\beta|+\varepsilon \log \lambda_{i}+\left|x_{i}-z_{i}\right|^{2}\right)\left|x_{i}-z_{i}\right|\right) \text {. }
$$

From these estimates, we deduce

$$
\begin{aligned}
& \frac{\partial \Psi_{\varepsilon}}{\partial \alpha_{i}}=O\left(|\beta|+\varepsilon \log \lambda_{i}+\frac{1}{\lambda_{i}}+\left|x_{i}-z_{i}\right|^{2}\right), \\
& \frac{\partial \Psi_{\varepsilon}}{\partial \lambda_{i}}=O\left(\frac{\varepsilon^{1+\sigma / 2}}{\lambda_{i}}\right) ; \quad \frac{\partial \Psi_{\varepsilon}}{\partial x_{i}}=O\left(\left|x_{i}-z_{i}\right|+\frac{1}{\lambda_{i}}\right) .
\end{aligned}
$$

By solving the system in $A, B$, and $C$, we find

$$
\left\{\begin{array}{l}
A_{i}=O\left(|\beta|+\varepsilon \log \lambda_{i}+\frac{1}{\lambda_{i}}+\left|x_{i}-z_{i}\right|^{2}\right), \\
B_{i}=O\left(\varepsilon^{1+\sigma / 2} \lambda_{i}\right) ; \quad C_{i}=O\left(\frac{\left|x_{i}-z_{i}\right|}{\lambda_{i}^{2}}+\frac{1}{\lambda_{i}^{3}}\right) .
\end{array}\right.
$$

Now, we can evaluate the right hand side in $\left(E_{\lambda_{i}}\right)$ and $\left(E_{x_{i}}\right)$,

$$
\begin{aligned}
& B_{i}\left\langle\frac{\partial^{2} \delta_{i}}{\partial \lambda_{i}^{2}}, \bar{v}\right\rangle+\sum_{j=1}^{4} C_{i j}\left\langle\frac{\partial^{2} \delta_{i}}{\partial x_{i}^{j} \partial \lambda_{i}}, \bar{v}\right\rangle=O\left(\left(\frac{\varepsilon^{1+\sigma / 2}}{\lambda_{i}}+\frac{\left|x_{i}-z_{i}\right|}{\lambda_{i}^{2}}+\frac{1}{\lambda_{i}^{3}}\right)\|\bar{v}\|\right), \\
& B_{i}\left\langle\frac{\partial^{2} \delta_{i}}{\partial \lambda_{i} \partial x_{i}}, \bar{v}\right\rangle+\sum_{j=1}^{4} C_{i j}\left\langle\frac{\partial^{2} \delta_{i}}{\partial x_{i}^{j} \partial x_{i}}, \bar{v}\right\rangle=O\left(\left(\varepsilon^{1+\sigma / 2} \lambda_{i}+\left|x_{i}-z_{i}\right|+\frac{1}{\lambda_{i}}\right)\|\bar{v}\|\right),
\end{aligned}
$$


where

$$
\left\|\frac{\partial^{2} P \delta_{i}}{\partial \lambda_{i}^{2}}\right\|=O\left(\frac{1}{\lambda_{i}^{2}}\right) ; \quad\left\|\frac{\partial^{2} P \delta_{i}}{\partial x_{i} \partial \lambda_{i}}\right\|=O(1) ; \quad\left\|\frac{\partial^{2} P \delta_{i}}{\partial x_{i}^{2}}\right\|=O\left(\lambda_{i}^{2}\right) .
$$

Now, we consider a point $\left(z_{1}, z_{2}\right) \in \partial S_{+}^{4} \times \partial S_{+}^{4}$ such that $z_{1}$ and $z_{2}$ are nondegenerate critical points of $K_{1}$. We set

$$
\frac{1}{\lambda_{i}}=\varepsilon \frac{S_{4}}{16 c_{3}} K\left(z_{i}\right)\left(\frac{\partial K}{\partial v}\left(z_{i}\right)\right)^{-1}\left(1+\zeta_{i}\right) ; \quad x_{i}=z_{i}+\xi_{i},
$$

where $\zeta_{i} \in \mathbb{R}$ and $\left(\xi_{1}, \xi_{2}\right) \in \mathbb{R}^{3} \times \mathbb{R}^{3}$ are assumed to be small.

Using (28) and these changes of variables, $\left(E_{\alpha_{i}}\right)$ becomes

$$
\beta_{i}=V_{\alpha_{i}}(\varepsilon, \beta, \zeta, \xi)=O\left(\beta^{2}+\varepsilon|\log \varepsilon|+|\xi|^{2}\right) .
$$

Also, we use (30), we have

$$
\begin{aligned}
\frac{2 c_{3}}{\lambda_{i}^{2}} \frac{\partial K}{\partial v}\left(z_{i}+\xi_{i}\right)+\frac{\varepsilon K\left(z_{i}+\xi_{i}\right) S_{4}}{8 \lambda_{i}} & \frac{\varepsilon^{2} S_{4}^{2} K\left(z_{i}\right)^{2}}{128 c_{3}}\left(\frac{\partial K}{\partial v}\left(z_{i}\right)\right)^{-2}\left(1+2 \zeta_{i}\right)\left(-\frac{\partial K}{\partial v}\left(z_{i}\right)+D^{2} K\left(z_{i}\right)\left(e_{4}, \xi_{i}\right)\right) \\
& +\frac{\varepsilon^{2} S_{4}^{2} K\left(z_{i}\right)^{2}}{128 c_{3}}\left(\frac{\partial K}{\partial v}\left(z_{i}\right)\right)^{-1}\left(1+\zeta_{i}\right)+O\left(\varepsilon^{2}\left(\zeta_{i}^{2}+\left|\xi_{i}\right|^{2}\right)\right) \\
= & -\frac{\varepsilon^{2} S_{4}^{2} K\left(z_{i}\right)^{2}}{128 c_{3}}\left(\frac{\partial K}{\partial v}\left(z_{i}\right)\right)^{-1} \zeta_{i} \\
& +\frac{\varepsilon^{2} S_{4}^{2} K\left(z_{i}\right)^{2}}{128 c_{3}}\left(\frac{\partial K}{\partial v}\left(z_{i}\right)\right)^{-2} D^{2} K\left(z_{i}\right)\left(e_{4}, \xi_{i}\right) \\
& +O\left(\varepsilon^{2}\left(\zeta_{i}^{2}+\left|\xi_{i}\right|^{2}\right)\right)
\end{aligned}
$$

Combining this with (31), then $\left(E_{\lambda_{i}}\right)$ becomes

$$
\begin{aligned}
-\zeta_{i}+\left(\frac{\partial K}{\partial v}\left(z_{i}\right)\right)^{-1} D^{2} K_{1}\left(z_{i}\right)\left(e_{4}, \xi_{i}\right) & =V_{\lambda_{i}}(\varepsilon, \beta, \zeta, \xi) \\
& =O\left(\varepsilon|\log \varepsilon|+|\beta|^{2}+\zeta_{i}^{2}+|\xi|^{2}\right) .
\end{aligned}
$$

Using (32), (33), and (36), $\left(E_{x_{i}}\right)$ is equivalent to

$$
D^{2} K_{1}\left(z_{i}\right) \xi_{i}=V_{x_{i}}(\varepsilon, \beta, \zeta, \xi)=O\left(\varepsilon^{1 / 2}+|\beta|^{2}+|\zeta|^{2}+|\xi|^{2}\right) .
$$

Observe that the functions $V_{\alpha_{i}}, V_{\lambda_{i}}$, and $V_{x_{i}}$ are smooth.

We can also write the system as

$$
\left\{\begin{array}{l}
\beta=V(\varepsilon, \beta, \zeta, \xi) \\
L(\zeta, \xi)=W(\varepsilon, \beta, \zeta, \xi)
\end{array}\right.
$$


where $L$ is a fixed linear operator on $\mathbb{R}^{8}$ defined by

$$
\begin{aligned}
L(\zeta, \xi)= & \left(-\zeta_{1}+\left(\frac{\partial K}{\partial v}\left(z_{1}\right)\right)^{-1} D^{2} K_{1}\left(z_{1}\right)\left(e_{4}, \xi_{1}\right) ;-\zeta_{2}+\left(\frac{\partial K}{\partial v}\left(z_{2}\right)\right)^{-1} D^{2} K_{1}\left(z_{2}\right)\left(e_{4}, \xi_{2}\right)\right. \\
& \left.D^{2} K_{1}\left(z_{1}\right) \xi_{1} ; D^{2} K_{1}\left(z_{2}\right) \xi_{2}\right)
\end{aligned}
$$

and $V, W$ are smooth functions satisfying

$$
\left\{\begin{array}{l}
V(\varepsilon, \beta, \zeta, \xi)=O\left(\varepsilon^{1 / 2}+|\beta|^{2}+|\xi|^{2}\right) \\
W(\varepsilon, \beta, \zeta, \xi)=O\left(\varepsilon^{\frac{1}{2}}+|\beta|^{2}+|\zeta|^{2}+|\xi|^{2}\right) .
\end{array}\right.
$$

Now, by an easy computation, we see that the determinant of the linear operator $L$ is not 0 . Hence $L$ is invertible, and according to Brouwer's fixed point theorem, there exists a solution $\left(\beta^{\varepsilon}, \zeta^{\varepsilon}, \xi^{\varepsilon}\right)$ of $(40)$ for $\varepsilon$ small enough, such that

$$
\left|\beta^{\varepsilon}\right|=O\left(\varepsilon^{1 / 2}\right) ; \quad\left|\zeta^{\varepsilon}\right|=O\left(\varepsilon^{1 / 2}\right) ; \quad\left|\xi^{\varepsilon}\right|=O\left(\varepsilon^{1 / 2}\right)
$$

Hence, we have constructed $m^{\varepsilon}=\left(\alpha_{1}^{\varepsilon}, \alpha_{2}^{\varepsilon}, \lambda_{1}^{\varepsilon}, \lambda_{2}^{\varepsilon}, x_{1}^{\varepsilon}, x_{2}^{\varepsilon}\right)$ such that $u_{\varepsilon}:=\sum \alpha_{i}^{\varepsilon} \delta_{\left(x_{i}^{\varepsilon}, \lambda_{i}^{\varepsilon}\right)}+\overline{v_{\varepsilon}}$, verifies (23)-(27). From Proposition $6, u_{\varepsilon}$ is a critical point of $I_{\varepsilon}$, which implies that $u_{\varepsilon}$ verify

$$
-\Delta u_{\varepsilon}+2 u_{\varepsilon}=K\left|u_{\varepsilon}\right|^{2-\varepsilon} u_{\varepsilon} \quad \text { in } S_{+}^{4}, \quad \partial u_{\varepsilon} / \partial v=0 \quad \text { on } \partial S_{+}^{4} .
$$

We multiply equation (41) by $u_{\varepsilon}^{-}=\max \left(0,-u_{\varepsilon}\right)$ and we integrate on $S_{+}^{4}$, we get

$$
\int_{S_{+}^{4}}\left|\nabla u_{\varepsilon}^{-}\right|^{2}+2 \int_{S_{+}^{4}}\left(u_{\varepsilon}^{-}\right)^{2}=\int_{S_{+}^{4}} K\left(u_{\varepsilon}^{-}\right)^{4-\varepsilon} .
$$

We know also from the Sobolev embedding theorem that

$$
\left|u_{\varepsilon}^{-}\right|_{4-\varepsilon}^{2}:=\left(\int_{S_{+}^{4}} K\left(u_{\varepsilon}^{-}\right)^{4-\varepsilon}\right)^{\frac{2}{4-\varepsilon}} \leq C\left\|u_{\varepsilon}^{-}\right\|^{2}
$$

Equations (42) and (43) imply that either $u_{\varepsilon}^{-} \equiv 0$, or $\left|u_{\varepsilon}^{-}\right|_{4-\varepsilon}$ is far away from zero. Since $m^{\varepsilon} \in M^{\varepsilon}$, we have $\left\|\overline{v_{\varepsilon}}\right\|<\nu_{0}$, where $v_{0}$ is a small positive constant (see the definition of $M_{\varepsilon}$ ). This implies that $\left|u_{\varepsilon}^{-}\right|_{4-\varepsilon}$ is very small. Thus, $u_{\varepsilon}^{-} \equiv 0$ for $\varepsilon$ small enough. Then $u_{\varepsilon}$ is a nonnegative function which satisfies (41). Finally, the maximum principle completes the proof of our theorem.

\section{Conclusion}

Thus it has been concluded that under some assumptions on the function $\mathrm{K}$, there exist solutions of the nonlinear problem $\left(S_{\varepsilon}\right)$ which are concentrated at two different points of the boundary. 


\section{Acknowledgements}

I would like to thank Deanship of Scientific Research at Taibah University for the financial support of this research project.

Received: 28 July 2016 Accepted: 14 November 2016 Published online: 25 November 2016

\section{References}

1. Diening, L, Harjulehto, $P$, Hasto, P, Ruzicka, M: Lebesgue and Sobolev Spaces with Variable Exponents. Lecture Notes in Mathematics, vol. 2011. Springer, Heidelberg (2011) MR2790542

2. Ambrosetti, A, Garcia Azorero, J, Peral, A: Perturbation of $\Delta u+u^{\frac{n+2}{n-2}}=0$, the scalar curvature problem in $\mathbb{R}^{n}$ and related topics. J. Funct. Anal. 165, 117-149 (1999)

3. Bahri, A, Coron, JM: The scalar curvature problem on the standard three dimensional spheres. J. Funct. Anal. 95, 106-172 (1991)

4. Bianchi, G, Pan, XB: Yamabe equations on half spheres. Nonlinear Anal. 37, 161-186 (1999)

5. Chang, SA, Yang, P: A perturbation result in prescribing scalar curvature on $S^{n}$. Duke Math. J. 64, 27-69 (1991)

6. Cherrier, P: Problèmes de Neumann non linéaires sur les variétés riemaniennes. J. Funct. Anal. 57, 154-207 (1984)

7. Escobar, J: Conformal deformation of Riemannian metric to scalar flat metric with constant mean curvature on the boundary. Ann. Math. 136, 1-50 (1992)

8. Escobar, J, Schoen, R: Conformal metrics with prescribed scalar curvature. Invent. Math. 86, 243-254 (1986)

9. Han, ZC, Li, YY: The existence of conformal metrics with constant scalar curvature and constant boundary mean curvature. Commun. Anal. Geom. 8, 809-869 (2000)

10. Hebey, E: The isometry concentration method in the case of a nonlinear problem with Sobolev critical exponent on compact manifolds with boundary. Bull. Sci. Math. 116, 35-51 (1992)

11. Li, YY: Prescribing scalar curvature on $S^{n}$ and related topics, Part I. J. Differ. Equ. 120, $319-410$ (1995); Part II. Existence and compactness. Comm. Pure Appl. Math. 49 437-477 (1996).

12. Ould Bouh, K: Blowing up of sign-changing solutions to a subcritical problem. Manuscr. Math. 146, 265-279 (2015)

13. Djadli, Z, Malchiodi, A, Ould Ahmedou, M: Prescribing the scalar and the boundary mean curvature on the three dimensional half sphere. J. Geom. Anal. 13, 233-267 (2003)

14. Ben Ayed, M, Ghoudi, R, Ould Bouh, K: Existence of conformal metrics with prescribed scalar curvature on the four dimensional half sphere. Nonlinear Differ. Equ. Appl. 19, 629-662 (2012)

15. Rey, O: The topological impact of critical points at infinity in a variational problem with lack of compactness: the dimension 3. Adv. Differ. Equ. 4, 581-616 (1999)

16. Bahri, A: An invariant for Yamabe-type flows with applications to scalar curvature problems in high dimension. A celebration of J. F. Nash jr. Duke Math. J. 81, 323-466 (1996)

17. Bahri, $A, L i, Y Y$, Rey, O: On a variational problem with lack of compactness: The topological effect of the critical points at infinity. Calc. Var. Partial Differ. Equ. 3, 67-94 (1995)

\section{Submit your manuscript to a SpringerOpen ${ }^{\circ}$ journal and benefit from:}

- Convenient online submission

Rigorous peer review

- Immediate publication on acceptance

- Open access: articles freely available online

- High visibility within the field

- Retaining the copyright to your article 